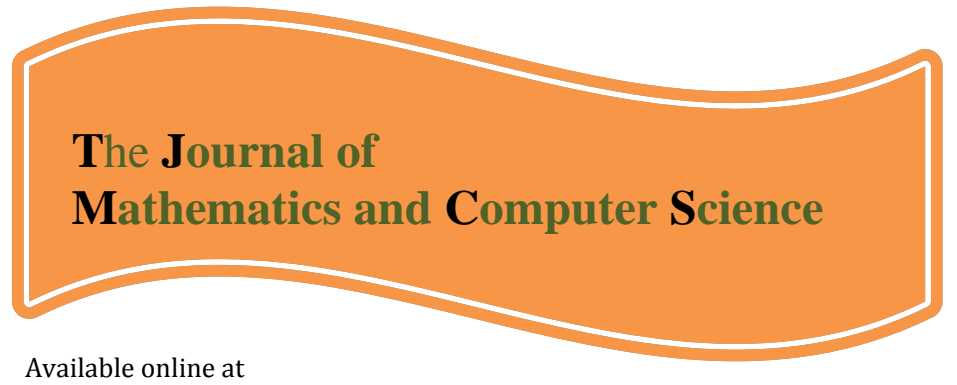

http://www.TJMCS.com

The Journal of Mathematics and Computer Science Vol .2 No.1 (2011) 27-36

\title{
Optimal Fuzzy Synchronization of Generalized Lorenz Chaotic Systems
}

\author{
Davood Babaei Pourkargar1, Mohammad Shahrokhi 2,* \\ Chemical and Petroleum Engineering Department, Sharif University of Technology, Babaie@che.sharif.ir \\ Chemical and Petroleum Engineering Department, Sharif University of Technology, Shahrokhi@sharif.ir
}

Received: September 2010, Revised: November 2010

Online Publication: January 2011

\begin{abstract}
In this article two identical generalized Lorenz systems have been synchronized by a fuzzy controller based on mamdani approach and stability of the proposed scheme has been established by the Lyapunov stability theorem. Controller parameters have been optimized by the genetic algorithm. Effectiveness of proposed method has been demonstrated through computer simulation.
\end{abstract}

Keywords: Fuzzy controller, Synchronization, Generalized Lorenz system, Genetic Algorithm.

\section{Introduction}

Synchronization of chaotic systems has received a significant attention, since Pecora and Carroll presented the chaos synchronization method for synchronizing two identical chaotic systems with different initial values [1]. Chaos synchronization can be applied widely in the fields of physics and

\footnotetext{
${ }^{1}$ M. Sc. Student, Chemical and Petroleum Engineering Department, Sharif University of Technology

$2,{ }^{*}$ Corresponding author, Professor of Chemical and Petroleum Engineering Department, Sharif University of Technology
} 
engineering systems, such as power converters, chemical reactions, biological systems, information processing, and especially in secure communication [2-6]. So far, different techniques and methods have been proposed to achieve chaos synchronization such as impulsive control [7,8], adaptive control $[9,10]$, sliding mode control [11-14], fuzzy control [15], optimal control [16], backstepping control [18,19], and so on. The Lorenz system is one of the paradigms of chaos, because it exhibits a wide variety of nonlinear dynamics phenomena such as bifurcations and chaos. Recently Lu et al. [20] have proposed the generalized chaotic system which includes the Lorenz and the Chen systems. The solution bounds of generalized Lorenz chaotic system (GLCS) are investigated based on the time-domain approach [21]. In that paper, the synchronization of two identical GLCSs with unknown parameters has been considered.

A fuzzy control system is a control system based on fuzzy logic [22]. Fuzzy logic is widely used in machine control and recently for chaos control and synchronization.

The genetic algorithm (GA) is a search heuristic that mimics the process of natural evolution. This algorithm is usually used to generate solutions to optimization and search problem. To optimize the controller parameters, different optimization techniques including genetic algorithm can be used.

In this work two identical GLCSs have been synchronized by a fuzzy controller whose parameters have been optimized via the GA.

\section{Problem Statement}

Consider two identical generalized Lorenz chaotic systems as given below:

$$
\begin{aligned}
& \text { Master system } \\
& \left\{\begin{array}{l}
\dot{x}_{1}=\left(10+\frac{25}{29} k\right)\left(x_{2}-x_{1}\right) \\
\dot{x}_{2}=\left(28-\frac{35}{29} k\right) x_{1}+(k-1) x_{2}-x_{1} x_{3} \\
\dot{x}_{3}=\left(-\frac{8}{3}-\frac{k}{87}\right) x_{3}+x_{1} x_{2}
\end{array}\right.
\end{aligned}
$$

This nonlinear system shows chaotic behavior when $k \in[0,1]$. The following figures (Fig1, Fig2 and Fig3) show chaotic attractor for $(\mathrm{k}=0.5)$. 




Fig.1. Chaotic behavior ( $\mathrm{x}_{1}$ vs. $\mathrm{t}$ )



Fig.2. Chaotic attractor ( $\mathrm{x}_{1}$ vs. $\left.\mathrm{x}_{2}\right)$ 


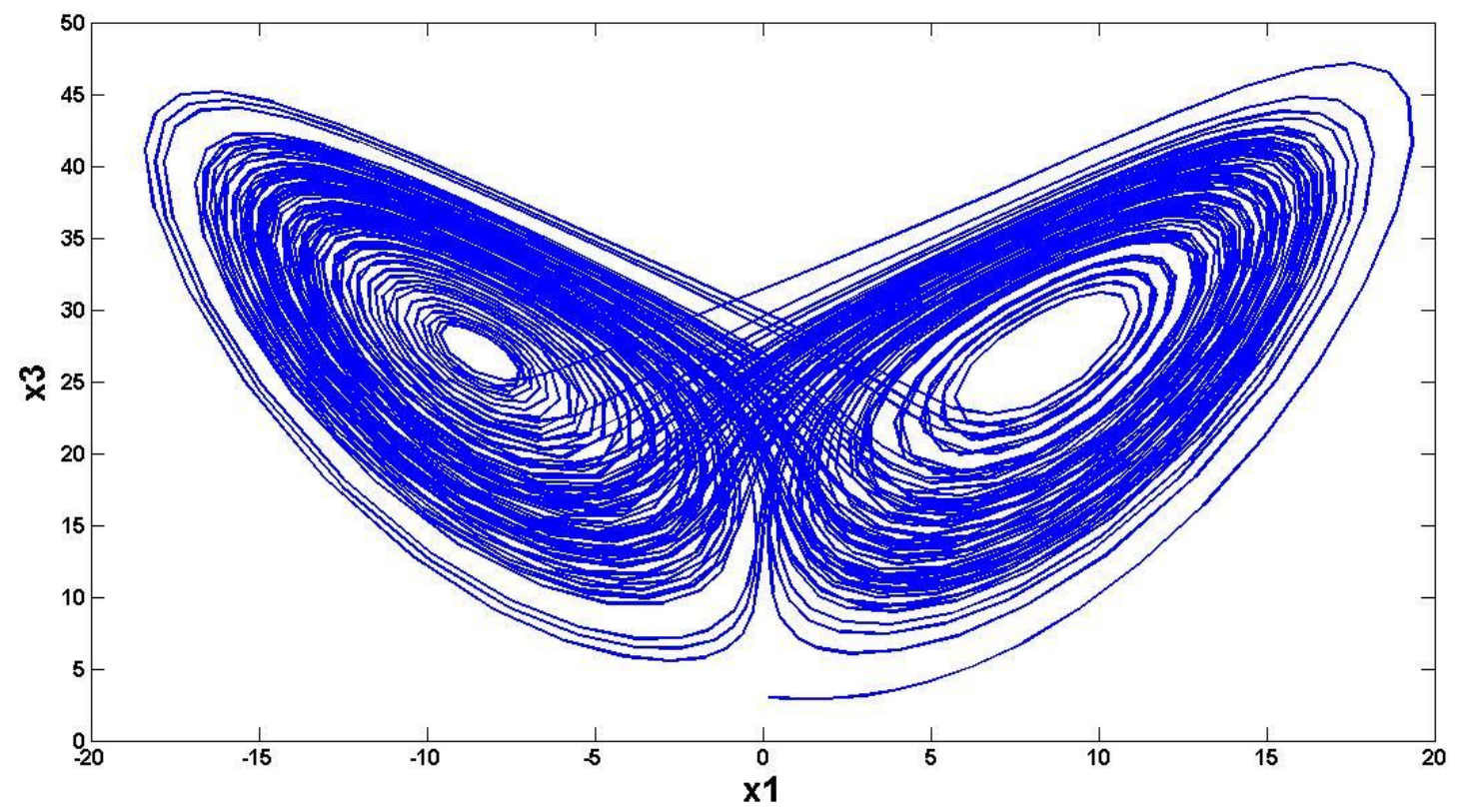

Fig.3. Chaotic attractor ( $\mathrm{x}_{1}$ vs. $\left.\mathrm{x}_{3}\right)$

Slave system

$$
\left\{\begin{array}{l}
\dot{y}_{1}=\left(10+\frac{25}{29} k\right)\left(y_{2}-y_{1}\right) \\
\dot{y}_{2}=\left(28-\frac{35}{29} k\right) y_{1}+(k-1) y_{2}-y_{1} y_{3}+u \\
\dot{y}_{3}=\left(-\frac{8}{3}-\frac{k}{87}\right) y_{3}+y_{1} y_{2}
\end{array}\right.
$$

Where $\mathrm{u}$ is the manipulated variable.

The objective is synchronizing the above chaotic systems. The synchronization errors have been defined as:

$\left\{\begin{array}{l}e_{1}=x_{1}-y_{1} \\ e_{2}=x_{2}-y_{2} \\ e_{3}=x_{3}-y_{3}\end{array}\right.$

The synchronization errors dynamics are given below: 


$$
\left\{\begin{array}{l}
\dot{e}_{1}=\left(10+\frac{25}{29} k\right)\left(e_{2}-e_{1}\right) \\
\dot{e}_{2}=\left(28-\frac{35}{29} k\right) e_{1}+(k-1) e_{2}+x_{1} x_{3}-y_{1} y_{3}+u \\
\dot{e}_{3}=\left(-\frac{8}{3}-\frac{k}{87}\right) e_{3}+e_{1} x_{2}+e_{2} y_{1}
\end{array}\right.
$$

$\mathrm{u}$ is chosen as below:

$$
u_{l}=x_{1} x_{3}-y_{1} y_{3}+u=u-u_{e q} \Rightarrow u=u_{l}+u_{e q}, u_{e q}=y_{1} y_{3}-x_{1} x_{3}
$$

If the first two equations of (4) were asymptotically stable or $\left(e_{1}, e_{2} \rightarrow 0\right)$ via the input variable $\left(u_{l}\right)$ then the last equation (4) reduces to:

$$
\dot{e}_{3}=\left(-\frac{8}{3}-\frac{k}{87}\right) e_{3}
$$

$e_{3}$ will be a stable internal error dynamics and $\left(e_{3} \rightarrow 0\right)$. So (4) reduced to

$$
\left\{\begin{array}{l}
\dot{e}_{1}=\left(10+\frac{25}{29} k\right)\left(e_{2}-e_{1}\right) \\
\dot{e}_{2}=\left(28-\frac{35}{29} k\right) e_{1}+(k-1) e_{2}+u_{l}
\end{array}\right.
$$

The above equation can be transformed to the following form:

$$
\left\{\begin{array}{l}
\frac{\bar{e}_{1}}{e_{1}}=\bar{e}_{2} \\
\overline{\bar{e}_{2}}=\left(10+\frac{25}{29} k\right)\left(27-\frac{6}{29} k\right) \bar{e}_{1}+\left(-11+\frac{4}{29} k\right) \bar{e}_{2}+u_{l}=a \bar{e}_{1}+b \bar{e}_{2}+u_{l}
\end{array}\right.
$$

By the following state transformation:

$$
\left[\begin{array}{l}
\bar{e}_{1} \\
\bar{e}_{2}
\end{array}\right]=\left[\begin{array}{cc}
\frac{1}{10+\frac{25 k}{29} k} & 0 \\
-1 & 1
\end{array}\right]\left[\begin{array}{l}
e_{1} \\
e_{2}
\end{array}\right] \Rightarrow\left\{\begin{array}{l}
\bar{e}_{1}=\frac{1}{10+\frac{25}{29} k} e_{1} \\
\bar{e}_{2}=-e_{1}+e_{2}
\end{array}\right.
$$

\section{Controller Design}

The described systems (1), (2) have been synchronized via the input variable $\left(\mathrm{u}_{\mathrm{l}}\right)$ that has been calculated by a fuzzy controller.

The fuzzy controller structure has been determined as follows:

$$
u_{l}=\operatorname{FLC}\left(\bar{e}_{1}, \bar{e}_{2}\right)
$$




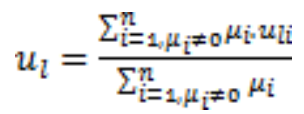

$\mu_{i}=\min \left(\mu_{R_{1}}\left(\bar{e}_{1}\right), \mu_{X_{2}}\left(\bar{e}_{2}\right)\right)$

The fuzzy membership functions are shown in Fig.2.

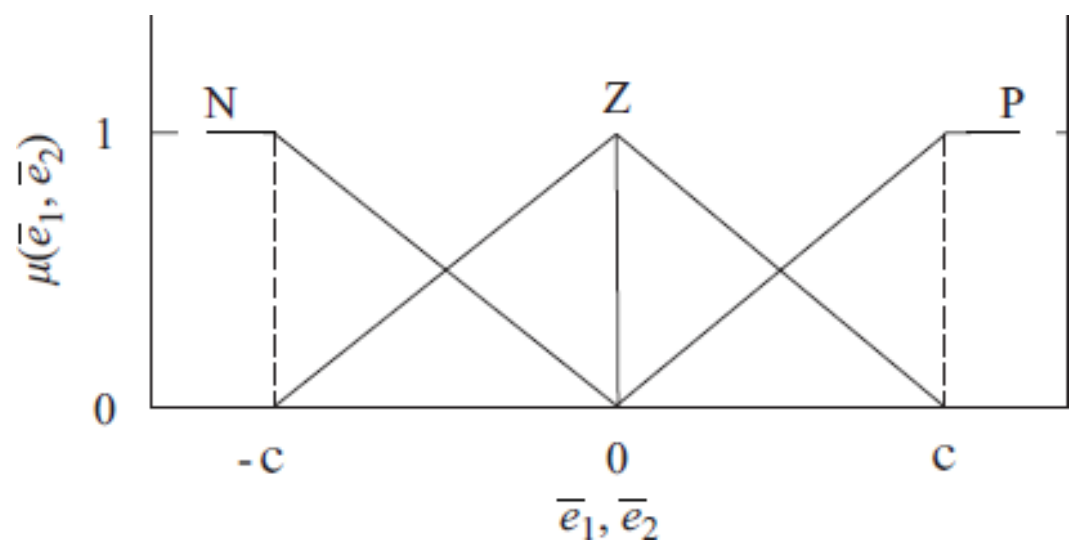

Fig.4 Membership functions

A typical fuzzy rule is as follows:

Rule $i:$ if $\left(\bar{e}_{1}\right.$ is $\left.X_{1}\right)$ \& $\left(\bar{e}_{2}\right.$ is $\left.X_{2}\right)$ then $u_{l i}=f_{i}\left(\bar{e}_{1}, \bar{e}_{2}\right)$

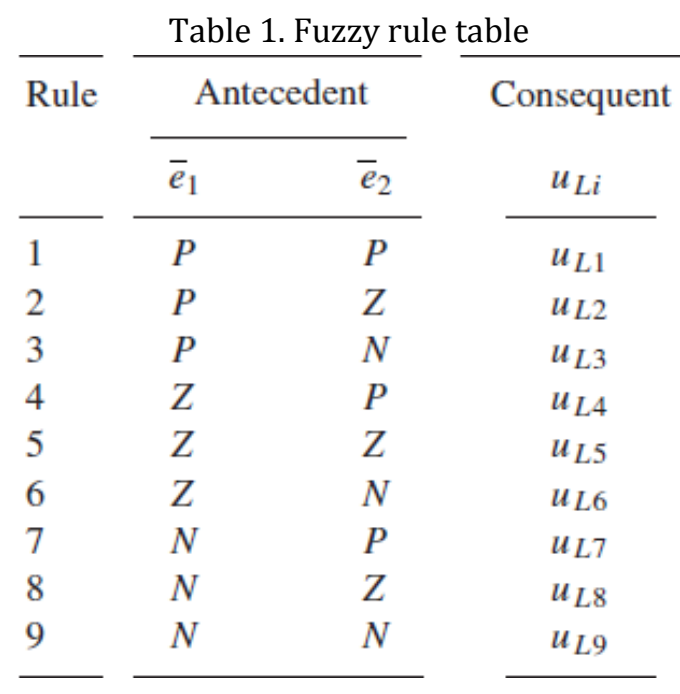

The rule table consequences of fuzzy module have been obtained based on a Lyapunov function. For controller design the following Lyapunov function has been used: 
$V=\frac{\left(\bar{\theta}_{1}^{2}+\bar{e}_{2}^{2}\right)}{2} \Rightarrow \dot{V}=\bar{e}_{1} \bar{e}_{1}+\bar{e}_{2} \bar{e}_{2}$

The control action has been selected such that the time derivative of the Lyapunov function becomes negative.

The consequents in Table.1 have been determined as follows:

Case1. $\bar{e}_{2}>0: \bar{e}_{2} \in P$

$$
\begin{gathered}
\bar{e}_{2}\left(\bar{e}_{1}+\dot{\bar{e}}_{2}\right)<0 \Rightarrow \bar{e}_{2}<-\bar{e}_{1} \Rightarrow \bar{e}_{2}=a \bar{e}_{1}+b \bar{e}_{2}+u_{l}<-\bar{e}_{1} \Rightarrow u_{l}<-(1+a) \bar{e}_{1}-b \bar{e}_{2} \\
u_{l}=-(1+a) \bar{e}_{1}-b \bar{e}_{2}-\alpha_{i}, \alpha_{i}>0 \\
u_{l i}=-(1+a) \bar{e}_{1}-b \bar{e}_{2}-\alpha_{i}, i=1,4,7
\end{gathered}
$$

Case2. $\bar{e}_{2}<0: \bar{e}_{2} \in N$

$$
\begin{gathered}
\bar{e}_{2}\left(\bar{e}_{1}+\dot{\bar{e}}_{2}\right)<0 \Rightarrow \dot{\bar{e}}_{2}>-\bar{e}_{1} \Rightarrow \dot{\bar{e}}_{2}=a \bar{e}_{1}+b \bar{e}_{2}+u_{l}>-\bar{e}_{1} \Rightarrow u_{l}>-(1+a) \bar{e}_{1}-b \bar{e}_{2} \\
u_{l}=-(1+a) \bar{e}_{1}-b \bar{e}_{2}+\alpha_{i}, \quad \alpha_{i}>0 \\
u_{l i}=-(1+a) \bar{e}_{1}-b \bar{e}_{2}+\alpha_{i}, i=3,6,9
\end{gathered}
$$

Case3. $\bar{e}_{2} \in Z, \bar{e}_{1} \in P$

$\bar{e}_{2}\left(\bar{e}_{1}+\overline{\bar{e}}_{2}\right)<0 \Rightarrow \bar{e}_{1}+\dot{\bar{e}}_{2}=-\operatorname{sgn}\left(e_{2}\right) \Rightarrow \dot{\bar{e}}_{2}=a \bar{e}_{1}+b \bar{e}_{2}+u_{l}<-\operatorname{sgn}\left(e_{2}\right)$

$\Rightarrow u_{l}<-a \bar{e}_{1}-b \bar{e}_{2}-\operatorname{sgn}\left(e_{2}\right) \Rightarrow u_{l}=-a \bar{e}_{1}-b \bar{e}_{2}-\operatorname{sgn}\left(e_{2}\right)-\alpha_{i}, \alpha_{i}>0$

$$
u_{12}=-a \bar{e}_{1}-b \bar{e}_{2}-\operatorname{sgn}\left(e_{2}\right)-\alpha_{2}
$$

Case4. $\bar{e}_{2} \in Z, \bar{e}_{1} \in N$

$\bar{e}_{2}\left(\bar{e}_{1}+\overline{\bar{e}}_{2}\right)<0 \Rightarrow \bar{e}_{1}+\dot{\bar{e}}_{2}=-\operatorname{sgn}\left(e_{2}\right) \Rightarrow \dot{\bar{e}}_{2}=a \bar{e}_{1}+b \bar{e}_{2}+u_{l}>-\operatorname{sgn}\left(e_{2}\right)$

$\Rightarrow u_{l}>-a \bar{e}_{1}-b \bar{e}_{2}-\operatorname{sgn}\left(e_{2}\right) \Rightarrow u_{l}=-a \bar{e}_{1}-b \bar{e}_{2}-\operatorname{sgn}\left(e_{2}\right)+\alpha_{i}, \alpha_{i}>0$

$$
u_{I 8}=-a \bar{e}_{1}-b \bar{e}_{2}-\operatorname{sgn}\left(e_{2}\right)+\alpha_{8}
$$

Case5. $\bar{e}_{2} \in Z, \bar{e}_{1} \in Z \Rightarrow u_{l 5}=0$

$$
u_{15}=0
$$




\section{Results}

Controller parameters have been optimized based on the following objective function which includes synchronization errors and a penalty term on the input:

$f=\int_{0}^{t}\left(\bar{e}_{1}^{2}+\bar{e}_{2}^{2}+u_{l}^{2}\right) d t$

The optimized controller parameters have been calculated via the GA as follows:

$b=0.18859$
$\alpha_{1}=17.15259$
$\alpha_{2}=18.01591$
$\alpha_{a}=15.68801$
$\alpha_{4}=28.42200$
$a_{6}=14.71820$
$a_{7}=3.07242$
$\alpha_{9}=24.65027$
$\alpha_{9}=29.92193$

For $\mathrm{k}=0.5$, and the initial states of the master system $(0.1,1.5,-0.6)$ and initial states of the slave system $(-0.3,-1,4)$, the synchronization errors have been shown in Fig.5. As can be seen, all errors have converged to zero and the two chaotic systems have been synchronized. 
Davood Babaei Pourkargar, Mohammad Shahrokhi / TJMCS Vol .2 No.1 (2011) 27-36

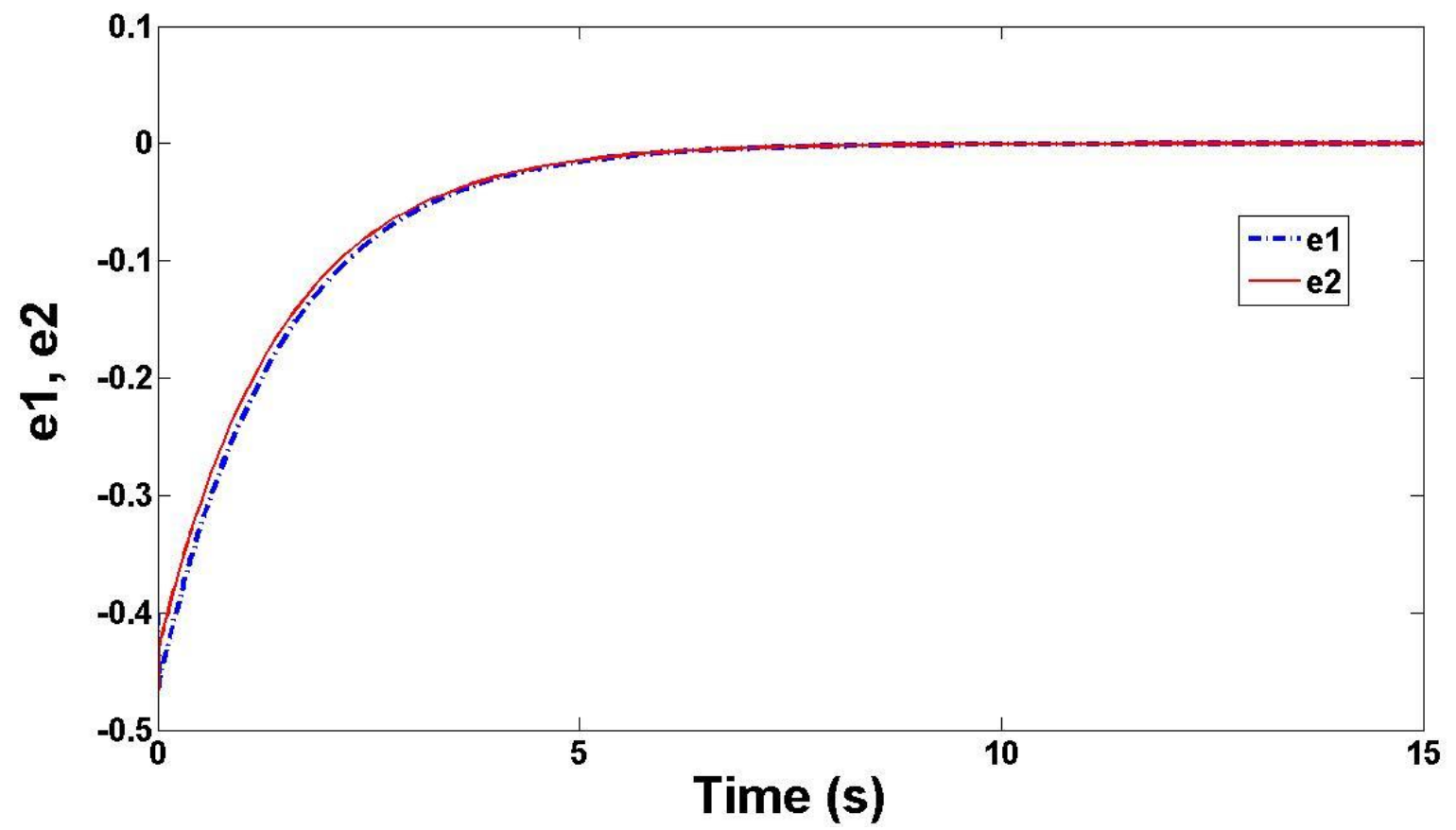

Fig.5a. First and second state synchronization errors

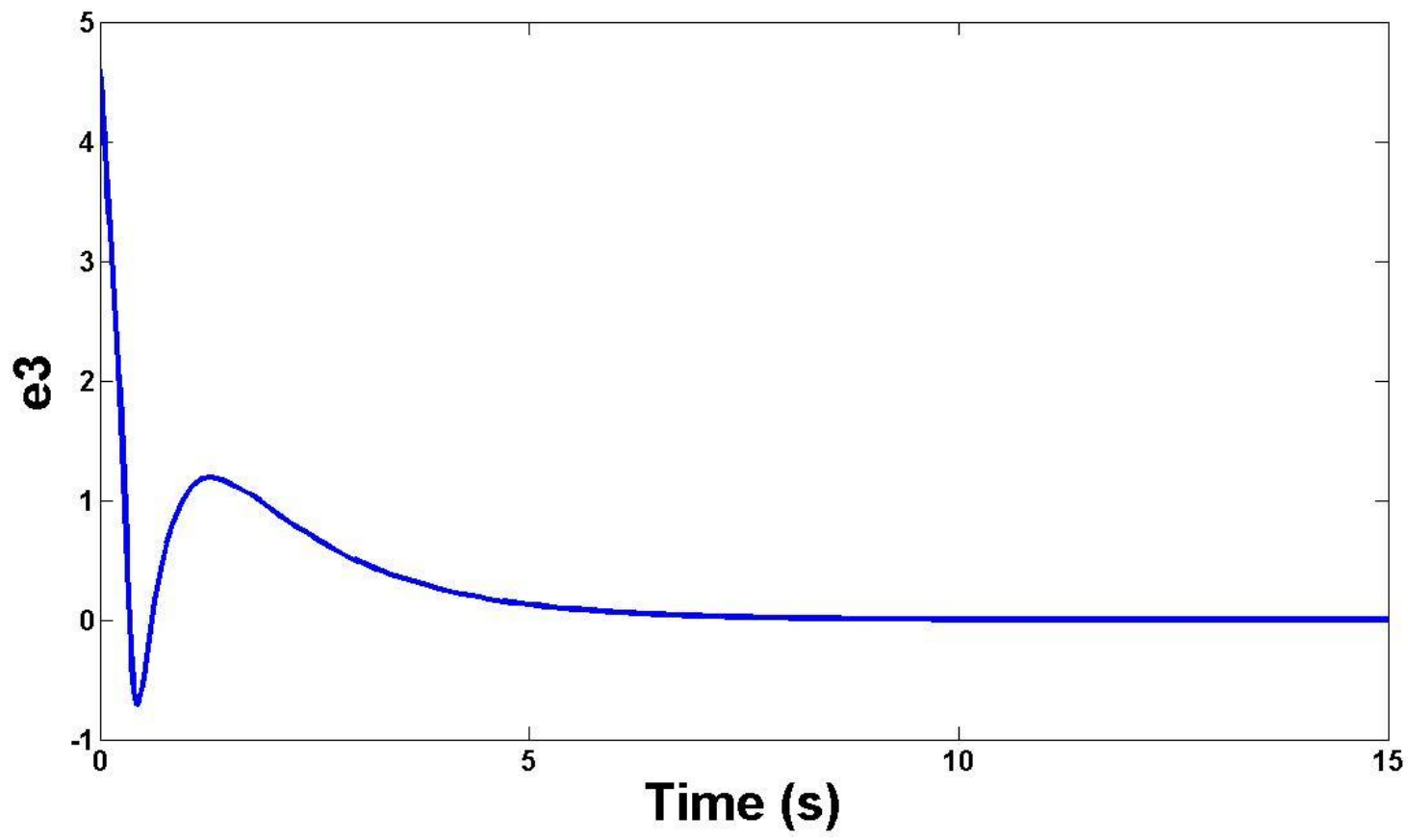

Fig.5b. Third state synchronization error 


\section{Conclusion}

In this paper two identical generalized Lorenz systems have been synchronized by a fuzzy controller based on mamdani approach. Stability of the proposed scheme has been established by the Lyapunov stability theorem. Then controller parameters have been optimized via the genetic algorithm. Simulation results show the effectiveness of proposed method.

\section{References}

[1] L.M. Pecora, T.L. Carroll, Synchronization in chaotic systems, Phys. Rev. Lett. 64 (8) (1990) 821-824.

[2] V.V. Astakhov, V.S. Anishchenko, T. Kapitaniak, A.V. Shabunin, Synchronization of chaotic oscillators by periodic parametric perturbations, Physica D 109 (1997) 11-16.

[3] G. Chen, X. Dong, From Chaos to Order: Methodologies, Perspectives and Applications, World Scientific, Singapore, 1998.

[4] L.O. Chua, T. Yang, G.Q. Zhong, C.W. Wu, Adaptive synchronization of Chua's oscillators, Int. J. Bifurcation Chaos 6 (1) (1996) 189-201.

[5] E.E. Escultura, Dynamic modeling of chaos and turbulence, Nonlinear Anal. 63 (5-7) (2005) 519-532.

[6] J.Q. Fang, Y. Hong, G. Chen, Switching manifold approach to chaos synchronization, Phys. Rev. E 59 (1999) $2523-$ 2526.

[7] G. Feng, G. Chen, Adaptive control of discrete-time chaotic systems: a fuzzy control approach, Chaos Solitons Fractals $23(2005)$ 459-467.

[8] C.L. Kuo, T.H. Li, N. Guo, Design of a novel fuzzy sliding-mode control for magnetic ball levitation system, J. Intell. Robotic Syst. (2004), 1-22.

[9] K.Y. Lian, P. Liu, T.S. Chiang, C.S. Chiu, Adaptive synchronization design for chaotic systems via a scalar Driving signal, IEEE Trans. CircuitsSyst. I 49 (1) (2002) 17-27.

[10] T.L. Liao, Adaptive synchronization of two Lorenz systems, Chaos Solitons Fractals 9 (1998) 1555-1561.

[11] J. Lu, S. Zhang, Controlling Chen's chaotic attractor using backstepping design based on parameters identification, Phys. Lett. A 286 (2001) 145-149.

[12] A.H. Nayfeh, Applied Nonlinear Dynamics, Wiley, New York, 1995.

[13] J.H. Park, Synchronization of Genesio chaotic system via backstepping approach, Chaos Solitons Fractals 27 (2006) 1369-1375.

[14] H.N. Agiza, Chaos synchronization of Lü dynamical system, Nonlinear Anal. 58 (1-2) (2004) 11-20.

[15] C.S. Shieh, Nonlinear rule-based controller for missile terminal guidance, IEE Proc. Control Theory Appl. 150 (1) (2003) 45-48.

[16] J.E. Slotine, W. Li, Applied Nonlinear Control, Prentice-Hall, New Jersey, 1991.

[17] J.A.K. Suykens, P.F. Curran, J. Vandewalle, IEEE Trans. Circuits Syst. I 44 (10) (1997) 891-904.

[18] F. Takeo, Chaos and hypercyclicity for solution semigroups to some partial differential equations, Nonlinear Anal. 63 (5-7) (2005) 1943-1953.

[19] K. Tanaka, T. Ikeda, H.O. Wang, A unified approach to controlling chaos via LMI-based fuzzy control system design, IEEE Trans Circuits Syst. I 45 (1998) 1021-1040.

[20] C.Wang, S.S. Ge, Adaptive synchronization of uncertain chaotic systems via backstepping design, Chaos Solitons Fractals 12 (2001) 199-206.

[21] Y. Wang, Z.H. Guan, X. Wen, Adaptive synchronization for Chen chaotic system with fully unknown parameters, Chaos Solitons Fractals 19 (2004) 899-903.

[22] L.A. Zadeh, Fuzzy logic, IEEE Comput. 21 (4) (1988) 83-93. 